\title{
Chlorosis of Ogura-CMS Brassica rapa is due to down-regulation of genes for chloroplast proteins
}

\author{
Seok-Won Jeong $\cdot$ Hankuil Yi $\cdot$ Hayoung Song $\cdot$ Soo-Seong Lee $\cdot$ Youn-II Park $\cdot$ Yoonkang Hur
}

Received: 13 June 2017 / Revised: 19 June 2017 / Accepted: 20 June 2017

(C) Korean Society for Plant Biotechnology

\begin{abstract}
Cytoplasmic male sterility (CMS) is a maternally inherited trait leading to loss of the ability to produce fertile pollen and is extensively used in hybrid crop breeding. OguraCMS was originally generated by insertion of orf 138 upstream of atp 8 in the radish mitochondrial genome and transferred to Brassica crops for hybrid breeding. Gene expression changes by dysfunctional mitochondria in Ogura-CMS result in pollen developmental defects, but little is known about gene expression patterns in vegetative tissue. To examine the interaction between nuclear and organellar regulation of gene expression, microarray and subsequent gene expression experiments were conducted with leaves of $F_{1}$ hybrid Chinese cabbage derived from selfincompatible (SI) or Ogura-CMS parents (Brassica rapa ssp. pekinensis). Out of 24,000 genes deposited on a KBGP24K microarray, 66 genes were up-regulated and 26 genes were down-regulated by over 2.5 fold in the CMS leaves. Up-regulated genes included stress-response genes and mitochondrial protein genes, while genes for ascorbic acid biosynthesis and thylakoid proteins were down-regulated. Most of the major component genes for light reactions of photosynthesis were highly expressed in leaves of both SI and CMS plants, but most of the corresponding proteins were found to be greatly reduced in leaves of CMS plants, indicating posttranscriptional regulation. Reduction in thylakoid proteins and chlorophylls led to reduction in photosynthetic efficiency and chlorosis of OguraCMS at low temperatures. This research provides a foundation for studying chloroplast function regulated by mitochondrial signal and for using organelle genome introgression in molecular breeding.
\end{abstract}

S.-W. Jeong $\cdot$ H. Yi $\cdot$ H. Song $\cdot$ Y.-I. Park $\cdot$ Y. Hur $(\bowtie)$ Department of Biological Science, College of Biological Science and Biotechnology, Chungnam National University, Daejeon 34134, Korea

e-mail: ykhur@cnu.ac.kr

S.-S. Lee

BioBreeding Institute, Business Incubation, Chung-Ang University, Ansung, Gyonggi Province 17546, Korea
Keywords Ogura-CMS, microarray, chlorosis, Chinese cabbage, photosynthesis

\section{Introduction}

Cytoplasmic male sterility (CMS) is a maternally-inherited trait that produces either aborted or infertile pollen grains. CMS is a consequence of miscoordination between nuclear and cytoplasmic gene products from different origins (Aviv and Galun 1980). These changes are usually caused by mutations, rearrangements, and/or recombinations in the mitochondrial genome, but not by nuclear gene mutations (Carlsson and Glimelius 2011). At least 14 mitochondrial genes that induce CMS have been characterized in plants (Chase 2007; Kojima et al. 2010). CMS is a valuable tool for commercial production of hybrid seeds in crops (Pelletier and Budar 2007), and is an excellent subject for the study of anterograde and retrograde signaling (Fujii and Toriyama 2008).

Ogura-CMS, originally identified in wild radish (Raphamus sativus) (Ogura, 1968), is controlled by a mitochondrial orf138 locus that consists of two co-transcribed open reading frames: orf138 and $\operatorname{orfB}$ (also called atp8, encoding ATP synthase subunit 8) (Bonhomme et al. 1991; Bonhomme et al. 1992; Krishnasamy and Makaroff 1993; Grelon et al. 1994). Brassica napus that contains Ogura-type CMS was originally produced by protoplast fusion (Pelletier et al. 1983) and transferred to Chinese cabbage in the 1980s (Yamagishi and Bhat 2014). Its first $\mathrm{F}_{1}$ hybrid seeds were produced from the CMS lines (Ke et al. 1992); however, these seeds have not been widely used because $F_{1}$ plants showed a negative effect, chlorosis at low temperature (LT), instead of heterosis. To eliminate these undesirable effects, B. rapa breeders produced new hybrids by protoplast fusion and repeated backcrossing successful in B. napus and B. juncea (Yamagishi and Bhat 2014). 
To understand mechanisms of Ogura-CMS in B. rapa, omics approaches have been recently conducted. Using $B$. rapa $300 \mathrm{~K}$ microarray, Dong et al. (2013) analyzed genes specific for pollen development stage and concluded that the retrograde signal from Ogura-CMS mitochondria delays expression of large number of nuclear genes involved in pollen development. Wei et al. (2015) identified important miRNAs and their target genes in Ogura-CMS Chinese cabbage using several omics data. However, these two researches have focused on pollen development in floral buds and no omics approaches have been applied to dissect gene expression profiles in vegetative tissues, such as leaf of Ogura-CMS Chinese cabbage.

Mitochondrial influence on the nuclear gene expression is referred to as mitochondrial retrograde regulation (MRR) and it occurs in CMS lines via CMS-inducing genes (Carlsson and Glimelius 2011), making CMS a useful system to study MRR (Chase 2007). Chloroplastic retrograde signaling changes both nuclear (Fernández and Strand 2008; Liao et al. 2016; Woodson 2016) and mitochondrial gene expression (Liao et al. 2016). However, little is known about the regulation of chloroplast genes and nuclear genes for chloroplast proteins by mitochondrial retrograde signaling. Especially, the role of retrograde pathway specific for CMS has never been described for plant vegetative tissues.

Chinese cabbage (B. rapa ssp. pekinensis) is one of the most important leafy vegetables in Asia and exhibits strong heterosis. Application of Ogura-CMS to produce $F_{1}$ seeds in Chinese cabbage has a high economic potential in seed industry, once accompanying problems like chlorosis have been resolved. To understand chlorosis development in Ogura-CMS Chinese cabbage under LT, we have examined gene expression profiles using KBGP24K microarray and compared chloroplast gene expression and photosynthetic activity. We concluded that chloroplast function was greatly inhibited in Ogura-CMS leaves due to the reduction of chloroplast gene expression by dysfunctional mitochondria.

\section{Materials and Methods}

Plant materials

Chinese cabbages (B. rapa ssp. pekinensis) were $\mathrm{F}_{1}$ hybrids obtained using either SI or Ogura-CMS (CMS) in BioBreeding Institute, Korea. Seeds were sown in pots on Aug. 10 and 3 week seedlings were transplanted to bigger pots and field. At 10-leaf stage (Fig. 1A) before the exposure to low temperature (LT) (the mid-September), $7^{\text {th }}$ to $9^{\text {th }}$ leaves were
A

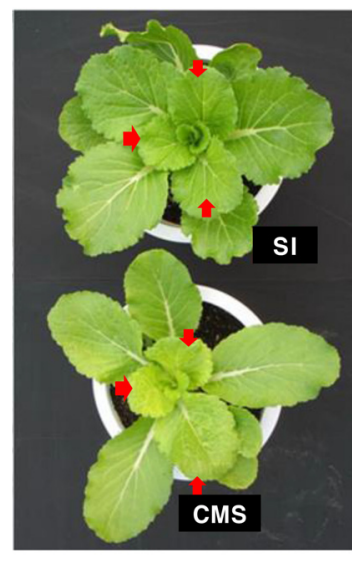

B

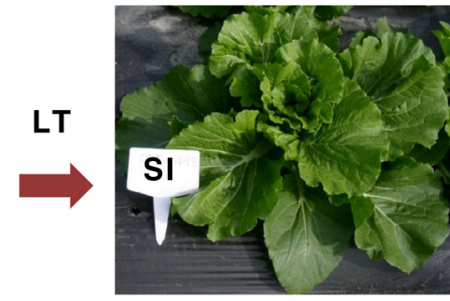

LT

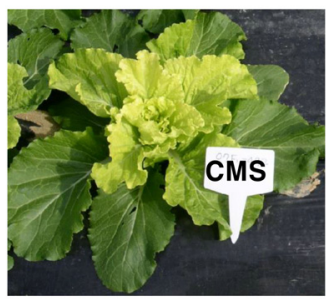

Fig. 1 Morphology of Chinese cabbage $F_{1}$ hybrids derived from SI or Ogura-CMS. A, Plants before low-temperature (LT) exposure (mid-September); CMS shows light chlorosis on $7^{\text {th }}$ to $9^{\text {th }}$ leaf, which are indicated by red arrows. B, Plants after LT exposure (mid-October); CMS shows severe chlorosis in young developing leaves

sampled from three individual plants and frozen in liquid nitrogen until use. Leaves from same developmental and environmental conditions were used for measurement of photosynthesis and western blot analysis.

\section{KBGP-24K microarray (Version 1)}

Using approximately 24,000 unigenes derived from EST analysis, oligomeric microarray was designed with 12 probes (six sense and six antisense) per gene (Lee et al. 2008). A set of 180,156 probes were designed, and duplicated in two separated block on a single chip. The 60 -nucleotide probes with $\mathrm{Tm}$ values of 75 to $85^{\circ} \mathrm{C}$ were synthesized on the slide using NimbleGen System (http://www.nimblegen. $\mathrm{com} /$ ). Random GC probes to monitor the hybridization efficiency and four corners to overlay the grid on the image were included.

Two biological replicates of total RNA were prepared from each plant sample and $10 \mu \mathrm{g}$ of total RNA were used for cDNA synthesis with Superscript Double-Stranded cDNA Synthesis Kit (Invitrogen, USA). Subsequent procedures for chip assay were followed as described (Lee et al. 2008). After normalization of probe intensity (Cy3 intensity), perfect match (PM) values of the six probes were used for selection of responsive genes. After removing genes with less than 1,000 PM value at all time points, genes specifically expressed or up-regulated in either tissue were selected and analyzed. 
Table 1 List of polyclonal antibodies used in this study. All antibodies were purchased from Agrisera Co. Ltd (Vännäs, Sweden)

\begin{tabular}{clll}
\hline $\begin{array}{c}\text { Gene } \\
\text { location }\end{array}$ & $\begin{array}{c}\text { Antibody } \\
\text { name }\end{array}$ & \multicolumn{1}{c}{$\begin{array}{c}\text { Protein } \\
\text { name }\end{array}$} & Classification \\
\hline Plastid & PsbA & Photosystem II protein D1 & PSII \\
& Cytf & Cytochrome f protein (PetA) of thylakoid Cytb6/f-complex & Electron transport \\
& PsaA & Photosystem I P700 chlorophyll a apoprotein A1 & PSI \\
& PsaC & Photosystem I iron-sulfur center & PSI \\
\hline Nucleus & LhcB1 & LHCII chlorophyll a/b binding protein 1-(1-5) & LHCII \\
& LhcB2 & LHCII type II chlorophyll a/b-binding protein & LHCI \\
& LhcA1 & PSI type I chlorophyll a/b-binding protein & LHCI \\
& LhcA2 & PSI type II chlorophyll a/b-binding protein &
\end{tabular}

Determination of chlorophyll fluorescence parameters

Changes in in vivo chlorophyll fluorescence were monitored through Xe-pulse amplitude modulated fluorometry (Walz, Germany) using cabbage leaf disc that were dark-adapted for $20 \mathrm{~min}$ before measurement. The $\mathrm{F}_{\mathrm{v}} / \mathrm{F}_{\mathrm{m}}$ value, which is an indicator for maximum PS II efficiency, was calculated as $\left(F_{m}-F_{0}\right) / F_{m}$, where $F_{v}$ is the dark-adapted variable fluorescence, $F_{m}$ is the maximum fluorescence and $F_{0}$ is the dark-adapted fluorescence. The actual quantum yield of PSII photochemistry in light-adapted cabbage leaf was calculated as $1-F / F_{m}{ }^{\prime}$, where $F$ is steady-state fluorescence and $F_{m}{ }^{\prime}$ is maximal fluorescence under illumination. Fluorescence quenching parameters were determined by qP, the coefficient of photochemical quenching, as defined by Schreiber et al. (1994), and NPQ (non-photochemical quenching: (Fm/Fm' - 1) during illumination at $800 \mu \mathrm{mol}$ photons $\left.\mathrm{m}^{-2} \mathrm{~s}^{-1}\right)$.

Determination of photosynthetic $\mathrm{O}_{2}$ evolution

Light-response curves of photosynthetic $\mathrm{O}_{2}$ evolution during illumination were determined with a leaf-disc $\mathrm{O}_{2}$ electrode (Oxygraph system, Hansatech, UK) in air with $5 \% \mathrm{CO}_{2}$ at $25^{\circ} \mathrm{C}$. Various irradiances (50 and $800 \mu \mathrm{mol}$ photons $\mathrm{m}^{-2} \mathrm{~s}^{-1}$ ) were provided using neutral density filters. The temperature was kept constant at $25^{\circ} \mathrm{C}$. The Chlorophyll content in leaf segments was determined from aqueous buffered $80 \%$ acetone extracts (25 mM Hepes, pH 7.5), as in Porra et al. (1989).

Analysis of proteins related to photosynthesis

Thylakoid protein components were measured immunochemically after isolation of the thylakoid membranes. Intact chloroplasts were isolated from leaves by homogenization
(Robinson and Barnett 1988). Thylakoid membranes were resuspended in $10 \mathrm{mM}$ Tricine- $\mathrm{NaOH}(\mathrm{pH} 7.0), 300 \mathrm{mM}$ sucrose, and $5 \mathrm{mM} \mathrm{MgCl}_{2}$. For protein gel blots, the membrane proteins were solubilized in $60 \mathrm{mM}$ Tris- $\mathrm{HCl}(\mathrm{pH} 7.8)$, $12 \%(\mathrm{w} / \mathrm{v})$ sucrose, $2 \%(\mathrm{w} / \mathrm{v})$ SDS, $1 \mathrm{mM}$ EDTA, and $58 \mathrm{mM}$ DTT. Protein gel electrophoresis was performed according to Laemmli (1970). The separated proteins were electrophoretically transferred to Immobilon-P membrane (Millipore). Chemiluminescence detection using antibodies was performed according to the manufacturer's instructions (Amersham Pharmacia Biotech.: ECL + Plus). Polyclonal antibodies raised against specific photosynthetic components were purchased from Agrisera Co (Vännäs, Sweden) (Table 1). The soluble protein contents were measured using BioRad protein assay reagents according to the manufacturer's instructions.

\section{Results}

Morphology of Chinese cabbage $\mathrm{F}_{1}$ hybrids derived from SI or Ogura-CMS

Since cultivated Chinese cabbage varieties are $F_{1}$ hybrids, we have focused on leaves of $F_{1}$ hybrids derived from SI and Ogura-CMS. As shown in Figure 1, CMS Chinese cabbage exhibited slight pale green before the exposure to LT for long period of time (the mid-September in Daejeon, Korea), but severe chlorosis in young developing leaves after the exposure to LT (the mid-October). These phenomena appear to be similar to that of previous work (Pelletier et al. 1983) and imply defective in photosynthetic efficiency or assembly of photosynthetic electron transport. All experiments were performed with slight pale green leaves (minor chlorosis). 
Table 2 Genes up-regulated in CMS by over 2.5-fold

\begin{tabular}{|c|c|c|c|}
\hline$B r \_$SEQ_ID & At_Locus & Gene Description & $\begin{array}{l}\text { Fold Change } \\
(\mathrm{CMS} / \mathrm{SI})\end{array}$ \\
\hline BRAS0001S00026533 & AT2G01520 & MLP328 (MLP-like protein 328) & 21.04 \\
\hline BRAS0001S00022245 & No_Hit & A09 sequence (3'UTR) & 13.67 \\
\hline BRAS0001S00003192 & AT3G08610 & Unknown (mitochondrial respiratory chain complex I) & 13.56 \\
\hline BRAS0001S00000806 & AT4G24420 & RNA-binding (RRM/RBD/RNP motifs) family protein & 9.38 \\
\hline BRAS0001S00010278 & AT2G07707 & Plant mitochondrial ATPase, F0 complex, subunit 8 protein & 7.36 \\
\hline BRAS0001S00022734 & No_Hit & Bra002978: Brassicarapaputativebeta-glucosidase41(LOC103844910) & 7.09 \\
\hline BRAS0001S00024268 & AT5G56010 & Bra035593: HSP90.3 (heat shock protein 81-3) & 6.44 \\
\hline BRAS0001S00015630 & AT4G24450 & GWD2; PWD (phosphoglucan, water dikinase) (involved in phosphorylation) & 6.29 \\
\hline BRAS0001S00017181 & AT3G48000 & Aldehyde dehydrogenase 2 & 5.69 \\
\hline BRAS0001S00017773 & AT2G07708 & Unknown protein (mitochondrion) & 5.40 \\
\hline BRAS0001S00004814 & AT1G70850 & MLP-LIKE PROTEIN 34 (MLP34) & 5.02 \\
\hline BRAS0001S00002171 & AT2G29460 & GSTU4 (Glutathione S-transferase 22) & 4.90 \\
\hline BRAS0001S00016599 & AT2G25140 & CLPB-M (Casein lytic proteinase B4)/HSP98.7 & 4.90 \\
\hline BRAS0001S00017434 & AT1G66130 & NAD(P)-binding Rossamann-fold superfamily protein & 4.64 \\
\hline BRAS0001S00018422 & AT3G49620 & DIN11 (Dark inducible 11) & 4.54 \\
\hline BRAS0001S00022560 & No_Hit & Bra030240 (no_hit_found) & 4.51 \\
\hline BRAS0001S00019384 & AT4G11890 & $\begin{array}{l}\text { ARCK1 (ABA- AND OSMOTIC-STRESS-INDUCIBLE RECEPTOR-LIKE } \\
\text { CYTOSOLIC KINASE1) }\end{array}$ & 4.43 \\
\hline BRAS0001S00023080 & No_Hit & Bra015764 (Brassicarapanucleolin2-like:LOC103832086) & 4.41 \\
\hline BRAS0001S00021743 & AT3G12580 & HSP70 & 4.20 \\
\hline BRAS0001S00000623 & AT5G56010 & HSP90.3/HSP81-3 & 4.16 \\
\hline BRAS0001S00006395 & AT2G40280 & S-adenosyl-L-methionine-dependent methyltransferases superfamily protein & 4.04 \\
\hline BRAS0001S00006011 & AT3G09350 & $\begin{array}{l}\text { FES1A (Encodes one of the } \\
\text { Arabidopsis orthologs of HspBP-1 and yeast Fes1p:Fes1A) }\end{array}$ & 4.01 \\
\hline BRAS0001S00014904 & AT5G24150 & SQE5/SQP1 (SQUALENE MONOOXYGENASE 5) & 3.91 \\
\hline BRAS0001S00004940 & AT2G46650 & CB5-C/CYTB5C (CYTOCHROME B5 ISOFORM C) & 3.73 \\
\hline BRAS0001S00010715 & AT3G12580 & HSP70 & 3.70 \\
\hline BRAS0001S00003420 & AT3G56060 & Glucose-methanol-choline (GMC) oxidoreductase family protein & 3.66 \\
\hline BRAS0001S00019692 & AT2G18860 & Bra038827; Syntaxin/t-SNARE family protein & 3.64 \\
\hline BRAS0001S00019491 & AT3G15210 & EFR4 (ETHYLENE RESPONSIVE ELEMENT BINDING FACTOR 4) & 3.61 \\
\hline BRAS0001S00019052 & AT3G22200 & GABA-T (GAMMA-AMINOBUTYRATE TRANSAMINASE) & 3.61 \\
\hline BRAS0001S00000758 & AT1G18540 & Ribosomal protein L6 family protein & 3.60 \\
\hline BRAS0001S00003941 & AT4G35160 & ASMT (N-ACETYLSEROTONIN O-METHYLTRANSFERASE) & 3.52 \\
\hline BRAS0001S00019993 & AT4G19840 & PP2-A1 (PHLOEM PROTEIN 2-A1) & 3.52 \\
\hline BRAS0001S00018407 & AT4G19645 & TRAM, LAG1 and CLN8 (TLC) lipid-sensing domain containing protein & 3.52 \\
\hline BRAS0001S00017767 & AT5G65070 & Agamous-like 69 (AGL69, FCL4, MAF4) & 3.48 \\
\hline BRAS0001S00013824 & AT5G56010 & Hsp90.3 (Hsp81.3) & 3.46 \\
\hline BRAS0001S00029134 & AT5G60200 & Dof-type transcription factor (DOF5.3) & 3.40 \\
\hline BRAS0001S00017206 & AT3G06880 & Transducin/WD40 repeat-like superfamily protein & 3.27 \\
\hline BRAS0001S00006213 & AT5G40240 & Nodulin MtN21-like transporter family protein & 3.17 \\
\hline BRAS0001S00026154 & AT4G01995 & Unknown & 3.16 \\
\hline BRAS0001S00029341 & AT4G17910 & Acyl transferase & 3.06 \\
\hline BRAS0001S00018291 & AT2G43650 & SAS10/C1D family protein (Embryodfective 2777) & 3.04 \\
\hline BRAS0001S00016302 & AT1G02820 & Late embryogenesis abundant 3 (LEA3) & 3.02 \\
\hline BRAS0001S00028858 & AT5G64040 & Encodes the only subunit of photosystem I located entirely in the thylakoid lumen & 2.99 \\
\hline BRAS0001S00023256 & AT3G22380 & Time for Coffee & 2.98 \\
\hline BRAS0001S00025328 & AT5G36230 & ARM repeat superfamily protein & 2.92 \\
\hline BRAS0001S00001224 & AT1G23260 & MMZ1/UEV1A (DNA damage response) & 2.88 \\
\hline BRAS0001S00001913 & AT5G02490 & HSP70-2 & 2.87 \\
\hline BRAS0001S00005028 & AT1G49600 & RNA-binding protein 47A (RBP47A) & 2.82 \\
\hline BRAS0001S00010541 & AT1G70830 & MLP-like protein 28 (MLP28) & 2.79 \\
\hline BRAS0001S00001764 & AT2G28000 & Chaperonin-60 alpha & 2.77 \\
\hline
\end{tabular}


Table 2 Continued

\begin{tabular}{cclcc}
\hline Br_SEQ_ID & At_Locus & & \multicolumn{1}{c}{ Gene Description } & $\begin{array}{c}\text { Fold Change } \\
\text { (CMS/SI) }\end{array}$ \\
\hline BRAS0001S00013300 & AT1G07790 & Histone 2B (HTB21) & 2.75 \\
BRAS0001S00015262 & AT5G59480 & Haloacid dehalogenase-like hydrolase (HAD) superfamily protein & 2.72 \\
BRAS0001S00010375 & AT5G09590 & Heat shock protein 70 (Hsc70-5) & 2.71 \\
BRAS0001S00014652 & AT1G76860 & Small nuclear ribonucleoprotein family protein (LSM3B) & 2.66 \\
BRAS0001S00006795 & AT1G75750 & GASA1 & 2.65 \\
BRAS0001S00011885 & AT3G61620 & Exonuclease RRP41 & 2.65 \\
BRAS0001S00011316 & AT5G40160 & Ankryin repeat protein EMB506 & 2.63 \\
BRAS0001S00008586 & AT1G06720 & P-loop containing nucleoside triphosphate hydrolases superfamily protein \\
BRAS0001S00018549 & AT3G04870 & PIGMENT DEFECTIVE EMBRYO 181 & 2.63 \\
BRAS0001S00004433 & AT3G19170 & Presequnce protease 1 & 2.61 \\
BRAS0001S00002521 & AT2G37990 & Ribosome biogenesis regulatory protein (RRS1) family protein \\
BRAS0001S00009108 & AT3G29200 & Chorismate mutase 1, chloroplast (CM1) & 2.57 \\
BRAS0001S00017531 & AT4G31210 & DNA topoisomerase family protein & 2.56 \\
BRAS0001S00011599 & AT4G23760 & COX19-like CHCG family protein & 2.56 \\
BRAS0001S00017175 & AT1G19730 & Thioredoxin-type r (TRX4) & 2.56 \\
BRAS0001S00003312 & AT3G48000 & Aldehyde dehydrogenase 2 & 2.51 \\
\hline
\end{tabular}

Table 3 Genes down-regulated in CMS over 2.5 fold

\begin{tabular}{|c|c|c|c|}
\hline$B r \_$SEQ ID & At_Locus & Gene Description & $\begin{array}{c}\text { Fold Change } \\
(\mathrm{CMS} / \mathrm{SI})\end{array}$ \\
\hline BRAS0001S00017904 & AT2G45790 & Cytoplasmic phosphomannomutase (ascorbate biosynthesis) & -4.97 \\
\hline BRAS0001S00017830 & ATCG00520 & YCF4 (Encodes a protein required for photosystem I assembly and stability) & -4.53 \\
\hline BRAS0001S00010846 & AT3G14210 & EPITHIOSPECIFIER MODIFIER 1, ESM1 & -4.51 \\
\hline BRAS0001S00000039 & AT3G27690 & LHC2 ( LIGHT-HARVESTING CHLOROPHYLL B-BINDING 2) & -4.13 \\
\hline BRAS0001S00013286 & AT3G14210 & ESM1 ( epithiospecifier modifier 1) & -4.06 \\
\hline BRAS0001S00000044 & AT5G48850 & SDI1 (sulphur deficincy-induced 1) & -3.30 \\
\hline BRAS0001S00009785 & AT1G52190 & NPF1.1 (nitrate transporter 1.11) & -3.29 \\
\hline BRAS0001S00024200 & No_Hit & Unknown & -3.26 \\
\hline BRAS0001S00005206 & AT1G75900 & Unknown & -3.25 \\
\hline BRAS0001S00001705 & AT1G15860 & Unknown & -3.19 \\
\hline BRAS0001S00022937 & AT1G74670 & GASA6 (GA-stimulated arabidopsis 6) & -2.94 \\
\hline BRAS0001S00018403 & AT1G25440 & BBX15 (B-box type zinc finger protein with CCT damain) & -2.89 \\
\hline BRAS0001S00002525 & AT5G02580 & Unknown & -2.81 \\
\hline BRAS0001S00006406 & AT2G44080 & ARL (ARGOS-LIKE) & -2.80 \\
\hline BRAS0001S00000344 & AT2G45960 & Aquaporin & -2.79 \\
\hline BRAS0001S00003430 & AT1G65310 & XTH17 (Xyloglucan-endotransglucosylase/hydrolase 17) & -2.77 \\
\hline BRAS0001S00006587 & AT5G37300 & WSD1 (wax ester synthase(WS) and diacylglycerol acyltransferase (DGAT) & -2.72 \\
\hline BRAS0001S00003214 & ATCG00530 & AYCF16 & -2.67 \\
\hline BRAS0001S00017873 & AT3G20370 & TRAF-like protein & -2.56 \\
\hline BRAS0001S00019530 & AT2G34620 & Mitochondrial tranascription termination factor family protein & -2.55 \\
\hline BRAS0001S00000993 & AT5G14030 & Transiocon-associated protein beta (TRAPB) family protein & -2.54 \\
\hline BRAS0001S00010320 & AT4G03560 & CCH1 (Calcium channel 1) & -2.53 \\
\hline BRAS0001S00008089 & AT5G19530 & ACL5 (Acaulis 5) & -2.53 \\
\hline BRAS0001S00021018 & AT1G02335 & GERMIN-LIKE PROTEIN SUBFAMILY 2 & -2.53 \\
\hline BRAS0001S00004281 & AT5G57800 & CER3 (Eceriferum 3)(similar to sterol desaturase family) & -2.51 \\
\hline BRAS0001S00019903 & AT1G28290 & AGP31 (Arabinogalactan protein 31)(vascular tissue function) & -2.50 \\
\hline
\end{tabular}

Analysis of differentially expressed genes (DEGs)

To identify DEGs in leaves of Ogura-CMS Chinese cabbage, transcriptomics experiment was carried out with KBGP24 oligomeric chips (Supplementary Table 1). Out of 24,000 genes, 66 genes and 26 genes were up-regulated and downregulated over 2.5 fold in the CMS, respectively (Table 2 and 3). Many up-regulated genes, such as HSP70s and 
$H S P 90$ s, are stress-related genes. Interestingly, genes encoding mitochondrial components were also up-regulated in CMS: mitochondrial respiratory chain complex I (BRAS0001S00 003192), mitochondrial ATPase subunit 8 (BRAS0001S00 010278) (Table 2). The highest up-regulated gene was BRAS 0001S00026533, which is related to a cis-cinnamic acid responsive gene (AT2G01520) in Arabidopsis thaliana. $A T 2 G 01520$ is a member of the major latex protein-like gene family, and plays a role in promoting vegetative growth or delaying flowering. On the other hand, down-regulated genes in CMS included a cytoplasmic phosphomannomutase -like gene (BRAS0001S00017904) and putative components for photosynthesis light reaction, such as YCF4 (BRAS000 1S00017830) and LHC2 (BRAS0001S00000039) (Table 3). These results suggest that protection for photosystems and light reaction efficiency could be greatly reduced in CMS Chinese cabbage. One more interesting finding was $E P I$ THIOSPECIFIER MODIFIER1 (ESM10) genes (BRAS0001 S00010846 and BRAS0001S00013286) were highly downregulated in CMS, altering glucosinolate hydrolysis and increasing insect feeding (Zhang et al. 2006).

Expression of photosynthesis-related genes

Expression of photosynthesis-related genes (encoding proteins for photosystem, electron transport and $\mathrm{CO}_{2}$ fixation) was strongly expressed in general, but there was no significant difference detected between SI and CMS Chinese cabbage at the transcript level (Table 4). Among LIGHT HARVESTING COMPLEX B (LHCB) 2.4 (= CYCLIN-DEPENDENT KINASE E1; CDKE1) paralogs, only BRAS0001S00000039 was highly up-regulated in SI compared to that of CMS.

To answer whether mitochondrial signal in Ogura-CMS affects expression of chloroplast and nucleus-encoded thylakoid proteins, expression levels of 8 proteins listed in Table 1 were examined by western blot analysis (Fig. 2). Except PsaA and PsaC, expressions of all other proteins showed

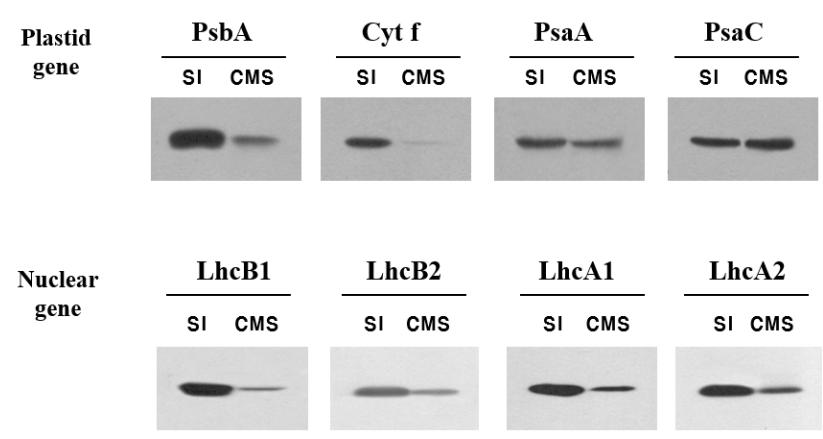

Fig. 2 Western blot analysis of thylakoid membrane components involved in the light reactions of photosynthesis

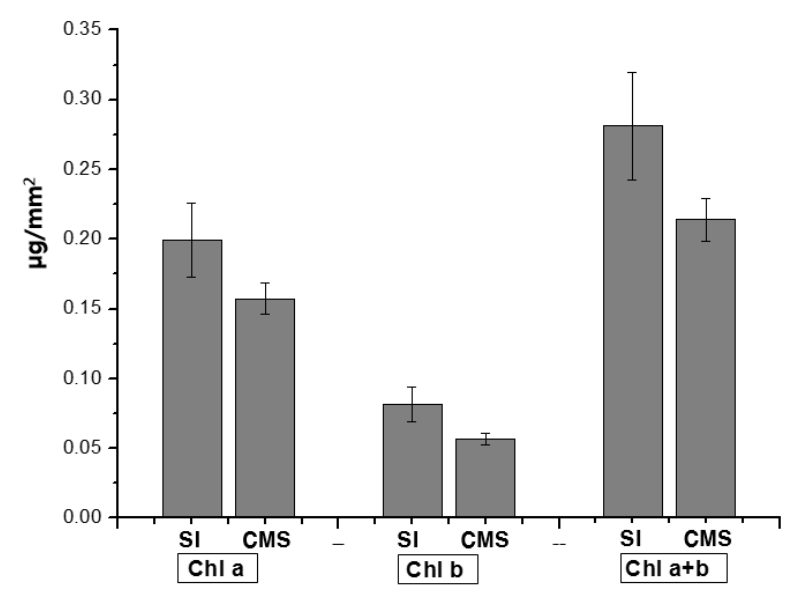

Fig. 3 Chlorophyll content of leaves from SI- and CMS-derived $\mathrm{F}_{1}$ hybrids of Chinese cabbage

a great reduction in CMS, suggesting that expression of these genes are regulated at the post-transcriptional levels. This result also revealed that mitochondria in Ogura-CMS affect plastid gene expression, along with nuclear gene expression.

Photosynthesis efficiency and chlorophyll content

Since protein levels associated with light reaction of photosynthesis were greatly reduced in CMS leaves (Fig. 2), we suspected that the pigment contents for photosynthetic reaction center would also be low in CMS-leaves. As shown in Figure 3, both chlorophyll a and b levels were low in CMSleaves, possibly related to the observation that Ogura-CMS Chinese cabbage develops chlorosis in LT (Fig. 1). Reduction in thylakoid proteins and chlorophyll $\mathrm{a} / \mathrm{b}$ has caused a lower photosynthetic efficiency in CMS-leaves (Fig. 4). It was found that both chlorophyll fluorescence parameter and $\mathrm{O}_{2}$ evolution were low in CMS-leaves. Yield expressed as electron flux through PSII was also low in CMS-leaves (Fig. 4A) and these results were consistent with the rate of oxygen evolution under the high light (Fig. 4B). The higher excitation pressure combined with lower non-photochemical quenching detected in CMS-leaves may be responsible for less resistance to high light in certain stress conditions, such as LT.

\section{Discussion}

CMS is important for hybrid breeding of crop plants and Ogura-CMS from wild radish can be an option for Chinese cabbage, which is an important leafy vegetable in Korea. However, $\mathrm{F}_{1}$ hybrids $B$. rapa derived from Ogura-CMS could not be widely used because $F_{1}$ plants did not show 
Table 4 Expression of photosynthesis-related genes from SI and CMS $\mathrm{F}_{1}$ Brassica rapa

\begin{tabular}{|c|c|c|c|c|c|c|c|}
\hline \multirow{2}{*}{ Classification } & \multirow{2}{*}{ At_Locus } & \multirow{2}{*}{ Gene Annotation } & \multirow{2}{*}{ BrSEQ_ID } & \multicolumn{2}{|c|}{ Probe Intensity } & \multicolumn{2}{|c|}{ Fold Change } \\
\hline & & & & SI & CMS & SI/CMS & $\mathrm{CMS} / \mathrm{SI}$ \\
\hline \multirow{37}{*}{ Photosystem } & \multirow{7}{*}{ AT3G27690 } & \multirow{7}{*}{$\begin{array}{l}\text { LIGHT HARVESTING COMPLEX B } \\
\text { (LHCB) } 2.4 \\
\text { :CYCLIN-DEPENDENT } \\
\text { KINASEE1(CDKE1)(LHCb2) }\end{array}$} & BRAS0001S00000039 & 4343 & 1051 & 4.1 & 0.2 \\
\hline & & & BRAS0001S00000036 & 57498 & 48062 & 1.2 & 0.8 \\
\hline & & & BRAS0001S00004511 & 858 & 831 & 1.0 & 1.0 \\
\hline & & & BRAS0001S00000149 & 58245 & 49668 & 1.2 & 0.9 \\
\hline & & & BRAS0001S00000425 & 50249 & 51813 & 1.0 & 1.0 \\
\hline & & & BRAS0001S00011198 & 45858 & 46917 & 1.0 & 1.0 \\
\hline & & & BRAS0001S00024982 & 45072 & 44977 & 1.0 & 1.0 \\
\hline & \multirow{2}{*}{ AT3G22370 } & \multirow[t]{2}{*}{ LHCb6 protein } & BRAS0001S00013783 & 37311 & 28983 & 1.3 & 0.8 \\
\hline & & & BRAS0001S00000558 & 33950 & 32016 & 1.1 & 0.9 \\
\hline & \multirow{2}{*}{ AT5G63610 } & \multirow[t]{2}{*}{ LHCa2 protein 2} & BRAS0001S00000423 & 33626 & 32622 & 1.0 & 1.0 \\
\hline & & & BRAS0001S00000424 & 23529 & 21477 & 1.1 & 0.9 \\
\hline & AT1G60950 & Putative $\mathrm{LHCa} 2$ protein & BRAS0001S00010799 & 1602 & 1452 & 1.1 & 0.9 \\
\hline & AT4G30650 & $\begin{array}{l}\text { Chlorophyll a/b-binding protein } \mathrm{CP} 26 \text { in } \\
\text { PS II }\end{array}$ & BRAS0001S00011564 & 55770 & 53536 & 1.0 & 1.0 \\
\hline & AT1G29910 & Chlorophyll $\mathrm{a} / \mathrm{b}$ binding protein & BRAS0001S00021229 & 55707 & 55473 & 1.0 & 1.0 \\
\hline & AT5G38420 & Putative chlorophyll a/b-binding protein & BRAS0001S00000056 & 55233 & 52843 & 1.0 & 1.0 \\
\hline & ATCG00740 & Chlorophyll $\mathrm{a} / \mathrm{b}$ binding protein & BRAS0001S00000031 & 54957 & 55997 & 1.0 & 1.0 \\
\hline & AT3G63160 & $\begin{array}{l}\text { Photosystem II chlorophyll-binding } \\
\text { protein PsbS }\end{array}$ & BRAS0001S00013419 & 54853 & 48328 & 1.1 & 0.9 \\
\hline & AT1G29930 & Chlorophyll $\mathrm{a} / \mathrm{b}$ binding protein & BRAS0001S00013518 & 50797 & 53893 & 0.9 & 1.1 \\
\hline & AT2G39730 & $\begin{array}{l}\text { Chlorophyll a/b-binding protein } \mathrm{CP} 26 \text { in } \\
\text { PS II }\end{array}$ & BRAS0001S00013654 & 49470 & 43664 & 1.1 & 0.9 \\
\hline & AT1G44575 & $\begin{array}{l}\text { PSI type III chlorophyll a/b-binding } \\
\text { protein }\end{array}$ & BRAS0001S00000421 & 49460 & 41222 & 1.2 & 0.8 \\
\hline & ATCG00530 & Chlorophyll $\mathrm{a} / \mathrm{b}$ binding protein & BRAS0001S00000217 & 45484 & 49544 & 0.9 & 1.1 \\
\hline & AT3G22120 & Chlorophyll a/b-binding protein-like & BRAS0001S00000385 & 43766 & 33051 & 1.3 & 0.8 \\
\hline & ATCG01100 & $\begin{array}{l}\text { Chlorophyll A-B binding protein / LHCI } \\
\text { type I (CAB) }\end{array}$ & BRAS0001S00011375 & 41492 & 31280 & 1.3 & 0.8 \\
\hline & AT1G29910 & Putative chlorophyll a/b-binding protein & BRAS0001S00000686 & 41411 & 26674 & 1.6 & 0.6 \\
\hline & AT2G07727 & LHCb3 chlorophyll $\mathrm{a} / \mathrm{b}$ binding protein & BRAS0001S00013469 & 41059 & 32760 & 1.3 & 0.8 \\
\hline & AT4G29350 & Chlorophyll $\mathrm{a} / \mathrm{b}$ binding protein & BRAS0001S00013494 & 39981 & 40065 & 1.0 & 1.0 \\
\hline & AT5G54770 & Chlorophyll a/b-binding protein-like & BRAS0001S00026135 & 38668 & 41453 & 0.9 & 1.1 \\
\hline & AT2G06520 & $\begin{array}{l}\text { Photosystem II chlorophyll-binding } \\
\text { protein PsbS }\end{array}$ & BRAS0001S00000186 & 37576 & 47164 & 0.8 & 1.3 \\
\hline & AT3G04120 & Chlorophyll a/b binding protein & BRAS0001S00000064 & 33412 & 32756 & 1.0 & 1.0 \\
\hline & AT2G15970 & Chlorophyll a/b-binding protein CP29 & BRAS0001S00000685 & 30857 & 28607 & 1.1 & 0.9 \\
\hline & AT1G54780 & $\begin{array}{l}\text { PSI type III chlorophyll a/b-binding } \\
\text { protein }\end{array}$ & BRAS0001S00000422 & 25078 & 26156 & 1.0 & 1.0 \\
\hline & AT1G08380 & Putative chlorophyll $\mathrm{a} / \mathrm{b}$ binding protein & BRAS0001S00014191 & 19691 & 22435 & 0.9 & 1.1 \\
\hline & AT3G62030 & Chlorophyll A-B binding family protein & BRAS0001S00015167 & 1523 & 2727 & 0.6 & 1.8 \\
\hline & AT1G55670 & Chlorophyll a/b-binding protein & BRAS0001S00023979 & 794 & 723 & 1.1 & 0.9 \\
\hline & AT5G54160 & Putative chlorophyll $\mathrm{a} / \mathrm{b}$ binding protein & BRAS0001S00010384 & 376 & 612 & 0.6 & 1.6 \\
\hline & AT4G10340 & Chlorophyll a/b-binding protein (cab-12) & BRAS0001S00000180 & 336 & 313 & 1.1 & 0.9 \\
\hline & AT1G61520 & $\begin{array}{l}\text { PSI type III chlorophyll a/b-binding } \\
\text { protein }\end{array}$ & BRAS0001S00013679 & 289 & 298 & 1.0 & 1.0 \\
\hline \multirow{17}{*}{$\begin{array}{l}\text { Electron } \\
\text { Transport }\end{array}$} & AT3G16670 & Cytochrome b561 & BRAS0001S00001778 & 27306 & 28235 & 1.0 & 1.0 \\
\hline & AT5G38420 & Cytochrome b559 & BRAS0001S00007312 & 11509 & 15551 & 0.7 & 1.4 \\
\hline & AT5G17870 & Cytochrome b5 & BRAS0001S00013366 & 10695 & 10876 & 1.0 & 1.0 \\
\hline & ATCG00480 & Cytochrome b5 & BRAS0001S00000053 & 5748 & 5853 & 1.0 & 1.0 \\
\hline & AT2G43560 & Cytochrome b6 & BRAS0001S00008540 & 4356 & 5801 & 0.8 & 1.3 \\
\hline & ATCG00630 & Putative cytochrome b5 & BRAS0001S00000068 & 4168 & 5772 & 0.7 & 1.4 \\
\hline & AT5G02380 & Cytochrome b5 & BRAS0001S00018351 & 4101 & 6001 & 0.7 & 1.5 \\
\hline & ATCG00140 & Cytochrome b5 & BRAS0001S00018217 & 3803 & 4762 & 0.8 & 1.3 \\
\hline & AT5G38410 & Cytochrome b-561D & BRAS0001S00009511 & 2633 & 1904 & 1.4 & 0.7 \\
\hline & AT2G02100 & Cytochrome b-561D & BRAS0001S00017536 & 2085 & 1718 & 1.2 & 0.8 \\
\hline & AT1G12090 & $\begin{array}{l}\text { Cytochrome b5 domain-containing } \\
\text { protein }\end{array}$ & BRAS0001S00010516 & 1252 & 1514 & 0.8 & 1.2 \\
\hline & AT1G08380 & Putative cytochrome b5 & BRAS0001S00005558 & 844 & 665 & 1.3 & 0.8 \\
\hline & AT4G10340 & Putative cytochrome b561 & BRAS0001S00005335 & 725 & 631 & 1.1 & 0.9 \\
\hline & AT1G29910 & Cytochrome b apoenzyme & BRAS0001S00022368 & 368 & 361 & 1.0 & 1.0 \\
\hline & AT3G56940 & Putative cytochrome b5 & BRAS0001S00011929 & 237 & 268 & 0.9 & 1.1 \\
\hline & AT5G18070 & $\begin{array}{l}\text { Plastocyanin-like domain-containing } \\
\text { protein }\end{array}$ & BRAS0001S00019135 & 3842 & 3333 & 1.2 & 0.9 \\
\hline & AT5G24340 & $\mathrm{Cu}^{2+}$ plastocyanin-likeprotein & BRAS0001S00010386 & 454 & 420 & 1.1 & 0.9 \\
\hline
\end{tabular}


Table 4 Continued

\begin{tabular}{|c|c|c|c|c|c|c|c|}
\hline \multirow{2}{*}{ Classification } & \multirow{2}{*}{ At_Locus } & \multirow{2}{*}{ Gene Annotation } & \multirow{2}{*}{$B r$ SEQ_ID } & \multicolumn{2}{|c|}{ Probe Intensity } & \multicolumn{2}{|c|}{ Fold Change } \\
\hline & & & & SI & CMS & SI/CMS & $\mathrm{CMS} / \mathrm{SI}$ \\
\hline \multirow{8}{*}{$\begin{array}{c}\mathrm{CO} 2 \\
\text { Fixation }\end{array}$} & \multirow{2}{*}{ AT1G20620 } & $\begin{array}{l}\text { Ribulose bisphosphate carboxylase } \\
\text { /oxygenase small subunit }\end{array}$ & BRAS0001S00019581 & 64284 & 63188 & 1.0 & 1.0 \\
\hline & & & BRAS0001S00019914 & 460 & 492 & 0.9 & 1.1 \\
\hline & AT5G38420 & $\begin{array}{l}\text { Ribulose bisphosphate carboxylase } \\
\text { /oxygenase small subunit }\end{array}$ & BRAS0001S00013358 & 59841 & 60723 & 1.0 & 1.0 \\
\hline & \multirow{2}{*}{ AT4G29350 } & $\begin{array}{l}\text { Ribulose bisphosphate carboxylase } \\
\text { /oxygenase small subunit }\end{array}$ & BRAS0001S00013535 & 59037 & 61972 & 1.0 & 1.0 \\
\hline & & & BRAS0001S00000087 & 1152 & 1373 & 0.8 & 1.2 \\
\hline & AT1G08380 & Ribulose bisphosphate carboxylase & BRAS0001S00000188 & 57299 & 60560 & 0.9 & 1.1 \\
\hline & AT1G07920 & $\begin{array}{l}\text { Ribulose bisphosphate carboxylase } \\
\text { /oxygenase small subunit }\end{array}$ & BRAS0001S00000032 & 49140 & 46726 & 1.1 & 1.0 \\
\hline & AT4G37210 & Ribulose bisphosphate carboxylase & BRAS0001S00013364 & 9254 & 9598 & 1.0 & 1.0 \\
\hline
\end{tabular}
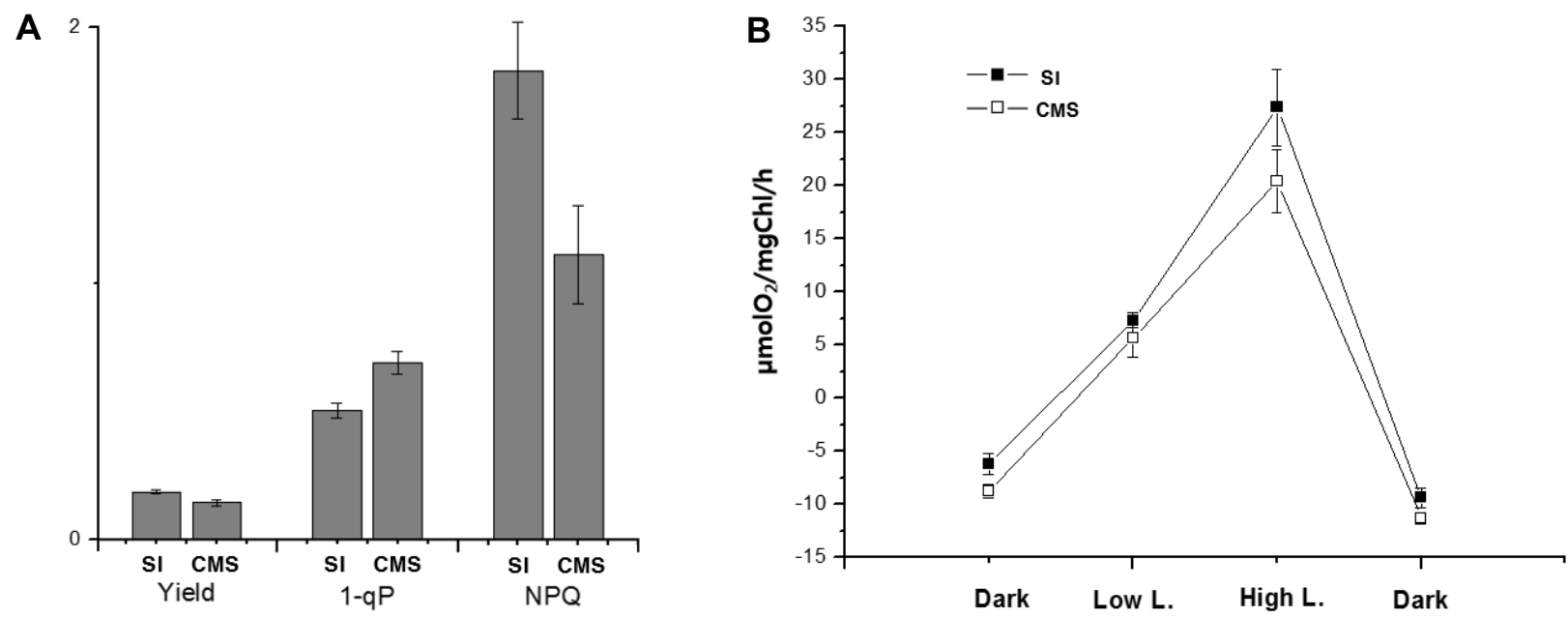

Fig. 4 Photosynthetic efficiencies of SI- and CMS-derived $\mathrm{F}_{1}$ hybrids: Chlorophyll fluorescence parameters $(\mathrm{A})$ and $\mathrm{O}_{2}$ evolution $(\mathrm{B})$. A, Electron flux through PSII (Yield), excitation pressure (1- qP), and non-photochemical quenching parameter (NPQ) in Chinese cabbage leaves under irradiance of $800 \mu \mathrm{mol}$ photons $\mathrm{m}^{-2} \mathrm{~s}^{-1}$. B, Light-response curves of photosynthetic $\mathrm{O}_{2}$ evolution in Chinese cabbage leaves.

heterosis but instead developed severe chlorosis under low temperature (Ke et al. 1992). This undesirable effect is due to the incompatibility between chloroplast and nucleus, and the problem could be overcome by chloroplast substitution, which involved somatic hybridization and repeated backcrossing to cabbage (Dey et al. 2013). With similar approaches being tried, more detailed understanding of the mechanism by which leaf chlorosis is induced can accelerate breeding efforts for Chinese cabbage.

From transcriptome analysis, it was found that DEGs in Ogura-CMS leaves (Table 2 and 3) are less obvious in gene numbers and fold changes in expression compared to those observed with male gametophyte (Dong et al. 2013; Wei et al. 2015). At the transcript level, most genes involved in photosynthesis were highly expressed in leaves of both SI- and CMS-derived F1 hybrid (Table 4 and Supplementary Table 1). However, accumulation of the proteins showed clear difference between two genotypes (Fig. 2), implying that expression of these genes are regulated at the posttranscriptional level. With reduced levels of thylakoid components, the amount of photosynthetic pigments and photosynthesis efficiency were also decreased (Fig. 3 and 4). In addition, heat stress-related protein genes were highly up-regulated in CMS-leaves (Table 2), suggesting the CMS mimics the effects of oxidative stress conditions. Particularly, down-regulation of phosphomannomutase gene in Ogura-CMS leaves implied that the protective capability of photosystem under oxidative stress is decreased. This gene is involved in ascorbate biosynthesis, which is related to high temperature tolerance (Hoeberichts et al. 2008). Ogura-CMS chloroplasts appear to be impaired in removal of excess energy absorbed by photosystems under high light (Fig. 4A), leading to the loss of chlorophyll pigments (Fig. 4B).

Both mitochondria and chloroplasts are important to maintain metabolic and energy homeostasis in the plant cell. Therefore, extensive researches on the interaction between 
Table 5 Expression of key retrograde signaling genes for chloroplast and mitochondria

\begin{tabular}{|c|c|c|c|c|}
\hline \multirow{2}{*}{$A t$ Locus } & \multirow{2}{*}{ Gene Annotation } & \multirow{2}{*}{ BrSEQ_ID } & \multicolumn{2}{|c|}{ PI (Probe intensity) } \\
\hline & & & SI & CMS \\
\hline \multirow[t]{6}{*}{ AT3G27690 } & LIGHT HARVESTING COMPLEX B (LHCB) 2.4 & BRAS0001S00000036 & 57,498 & 48,062 \\
\hline & & BRAS0001S00013452 & 49,690 & 35,900 \\
\hline & & BRAS0001S00000079 & 49,312 & 41,730 \\
\hline & & BRAS0001S00023696 & 34,142 & 21,332 \\
\hline & & BRAS0001S00000039 & 4,343 & 1,051 \\
\hline & & BRAS0001S00013388 & 1,329 & 1,195 \\
\hline \multirow[t]{2}{*}{ AT3G22370 } & ALTERNATIVE OXIDASE1a & BRAS0001S00000062 & 1,378 & 1,372 \\
\hline & & BRAS0001S00000137 & 1,364 & 1,375 \\
\hline \multirow[t]{2}{*}{ AT5G63610 } & CYCLIN-DEPENDENT KINASE E1 (CDKE1) & BRAS0001S00027374 & 3,788 & 3,714 \\
\hline & & BRAS0001S00004511 & 858 & 831 \\
\hline AT2G40220 & ABSCISIC ACID INSENSITIVE 4 (ABI4) & . & & . \\
\hline
\end{tabular}

these organelles have been carried out with respect to photosynthesis and respiration at physiological levels. But, only one paper (Liao et al. 2016) has mentioned that dysfunctional chloroplast is related to the up-regulation of mitochondrial gene expression in Arabidopsis. Our results is the first report for the chloroplast gene expression change by dysfunctional mitochondria, showing accumulation of chloroplast proteins can be regulated by mitochondrial signal.

Plant mutations responsible for mitochondrial dysfunction result in change of nuclear gene expression (Newton et al. 2004; Dong et al. 2013), and several candidate signals have suggested: redox sensors and signals, kinases/phosphatases, hormones, and other sensors (Rhoads 2011). Recently, key genes for retrograde signaling for chloroplast and mitochondria have been identified (Giraud et al. 2009; Blanco et al. 2014; Saha et al. 2016). The expression of these four key genes in our experiments using Chinese cabbage was not similar to Arabidopsis data (Table 5). Only one paralog (BRASO00 1S00000039) corresponding to Arabidopsis AT3G27690 ( $\mathrm{LHCb}$ ) was differentially expressed between SI- and CMS leaves. These results may suggest that retrograde signaling or organelle interaction is regulated at the protein level or different signaling components unique to species are used. Considering that finding of the best combination between nucleus and organelles is prerequisite for CMS-based breeding, molecular mechanisms associated with CMS need to be further elucidated.

\section{Acknowledgements}

This work was supported by Research Fund of Chungnam National University (CNU), Daejeon, Korea, to Yoonkang Hur (2016).

\section{References}

Aviv D, Galun E (1980) Restoration of fertility in cytoplasmic male sterile (CMS) Nicotiana Sylvestris by fusion with X-irradiated N. tabacum protoplasts. Theor Appl Genet 58: 121-127

Blanco NE, Guinea-Díaz M, Whelan J, Strand Å (2014) Interaction between plastid and mitochondrial retrograde signaling pathways during changes to plastid redox status. Philos Trans R Sos Lond B Biol Sci 369: 20130231

Bonhomme S, Budar F, Férault M, Pelletier G(1991) A 2.5 kb NcoI frgment of Ogura radish mitochondrial DNA is correlated with cytoplasmic male-sterilty in Brassica cybrids. Curr Genet 19: 121-127

Bonhomme S, Budar F, Lancelin D, Small I, Defrance MC, Pelletier G (1992) Sequence and transcript analysis of the Nco2.5 Ogura-specific fragment correlated with cytoplasmic male sterility in Brassica cybrids. Mol Gen Genet 235: 340-348

Carlsson J, Glimelius K (2011) Cytoplasmic male-sterility and nuclear encoded fertility restoration. Plant Mitochond 1: 469-491

Chase CD (2007) Cytoplasmic male sterility: a window to the world of plant mitochondrial-nuclear interactions. Trends Genet 23:81-90

Dey SS, Bhatia R, Sharma SR, Parkash C, Sureja AK (2013) Effects of chloroplast substituted Ogura male sterile cytoplasm on the performance of cauliflower (Brassica oleracea var. botrytis L.) F1 hybirds. Sci Hort 157:45-51

Dong X, Kim WK, Lim YP, Kim YK, Hur Y (2013) Ogura-CMS in Chinese cabbage (Brassica rapa ssp. pekinensis) cuases delayed expression of many nuclear genes. Plant Sci 199-200: 7-17

Fernández AP, Strand A (2008) Retrograd signaling and plant stress: plastid signals initiate cellular stress response. Curr. Opin. Plant Biol 11:509-513 
Fujii S, Toriyama K (2008) Genome barriers between nuclei and mitochondria exemplified by cytoplasmic male sterility. Plant Cell Physiol 49:1484-1494

Giraud E, Aken OV, Ho LH, Whelan J (2009) The transcriptional factor $\mathrm{ABI} 4$ is a regulator of mitochondrial retrograde expression of ALTERNATIVE OXIDASE1a. Plant Physiol 150:1286-1296

Grelon M, Budar F, Bonhomme S, Pelletier G (1994) Ogura cytoplasmic male-sterility (CNS)-associated orf138 is translated into a mitochondrial membrane polypeptide in male-sterile Brassica cybrids. Mol Gen Genet 243:540-547

Hoeberichts FA, Vaeck E, Kiddle G, Coppens E, van de Cotte B, Adamantidis A, Ormenese S, Foyer CH, Zabeau M, Inze D et al. (2008) A temperature sensitive mutation in the Arabidopsis thaliana phosphomannomutase gene disrupts protein glycosylation and triggers cell death. J Biol Chem 283:5708-5718

Ke GL, Zhao ZY, Song YZ, Zhang LG, Zhao LM (1992) Breeding of alloplasmic male sterile line cms3411-7 in Chinese cabbage (Brassica campestris L. ssp.pekinensis (lour) olsson) and its application. Acta Hortic Sinica. 19:333-340

Kojima H, Kazama T, Fujii S Toriyama K (2010) Cytoplasmic male sterility-associated orf79 is toxic to plant regeneration when expressed with mitochondrial targeting sequence of ATPase $\gamma$ subunit. Plant Biotechnol 27:111-114

Krishnasamy S, MakaroffCA (1993) Characterization of the radish mitochondrial orfB locus: possible relationship with male sterility in Ogura radish. Curr Genet 24:156-163

Laemmli BK (1970) Cleavage of structural proteins during the assembly of the head of bacteriophage T4. Nature 223: 680-685

Lee SC, Lim MH, Kim JA, Lee SI, Kim JS, Jin M, Kwon SJ, Mun JH, Kim YK, Kim HU, Hur Y, Park BS (2008) Transcriptome analysis in Brassica rapa under the abiotic stresses using Brassica 24K oligo microarray. Mol Cells 26:595-605

Liao JC, Hsieh WY, Tseng CC, Hsieh MH (2016) Dysfunctional chloroplasts up-regulate the expression of mitochondrial genes in Arabidopsis seedlings. Photosynth Res 127:151-159

Newton KJ, Gabay-Laughnan S, DePaepe R (2004) Mitochondrial mutations in plants. In: Day DA, Millar AH, Whelan J (Eds) Advances in Photosynthesis and Respiration, vol. 17. Kluwer Academic Publishers, Dordrecht, pp 121-142
Ogura H (1968) Studies of a new male-sterility in Japanese radish, with special reference to the utilization of this sterility towards the practical raising of hybrid seeds. Mem Fac Agric, Kagoshima Univ 6:39-78

Pelletier G, Budar F (2007) The molecular biology of cytoplasmically inherited male sterility and prospects for its engineering. Curr Opin Biotechnol 18:121-125

Pelletier G, Primard C, Vedel F, Chétrit P, Rémy R, Rousselle P, Renard M (1983) Intergeneric cytoplasmic hybridization in Cruciferae by protoplast fusion. Mol Gen Genet 191:244-250

Porra RJ, Thompson WA, Kriedemann PE (1989) Determination of accurate extinction coefficients and simultaneous equations for assaying chlorophylls a and $b$ extracted with four different solvents: verification of the concentration of chlorophyll standards by atomic absorption spectroscopy. Biochim Biophys Acta 975:384-394

Rhoads DM (2011) Plant mitochondrial retrograde regulation. Plant Mitochond 1:411-437

Robinson C, Barnett L K (1988) Isolation and analysis of chloroplasts; in Plant Molecular Biology, a Practical Approach, Shaw CH (ed.), pp 67-78, IRL Press, Washington

Saha B, Borovskii G, Panda SK (2016) Alternative oxidase and plant stress tolerace. Plant Signal Behav 11:e1256530

Schreiber U, Bilger W, Neubauer C (1994) Chlorophyll fluorescence as a nonintrusive indicator for rapid assessment of in vivo photosynthesis; in Ecophysiology of Photosynthesis, Schulze ED, Caldwell MM (eds), pp 49-70, Springer-Verlag, Berlin

Wei X, Zhang X, Yao Q, Yuan Y, Li X, Wei F, Zhao Y, Zhang Q, Wang Z, Jiang W, Zhang X (2015) The miRNAs and their regulatory networks responsible for pollen abortion in OguraCMS Chinese cabbage revealed by high-throughput sequencing of miRNAs, degradomes, and transcriptomes. Front Plant Sci 6:894

Woodson JD (2016) Chloroplast quality control - balancing energy production and stress. New Phytol 212:36-41

Yamagishi H, Bhat SR (2014) Cytoplamsic male sterility in Brassicacae crops. Breeding Sci 64:38-47

Zhang Z, Ober JA, Kliebenstein DJ (2006) The gene controlling the quantitative trait locus EPITHIOSPECIFIER MODIFIER I alters glucosinolate hydrolysis and insect resistance in Arabidopsis. Plant Cell 18:1524-1536 